\title{
Die idiopathische pulmonale Fibrose jenseits der Lunge: Krankheitsmechanismen verstehen, um Diagnose und Therapie zu verbessern
}

\author{
Fabrizio Luppi $^{\mathrm{a}} \quad$ Meena Kalluri $^{\mathrm{b}}$ Paola Faverio $^{\mathrm{a}}$ Michael Kreuter $^{\mathrm{c}}$ Giovanni Ferrarab, $\mathrm{d}$
}

${ }^{a}$ Respiratory Unit, University of Milano Bicocca, S. Gerardo Hospital, ASST Monza, Monza, Italien; ${ }^{b}$ Division of Pulmonary Medicine, Department of Medicine, University of Alberta, 3-134 Clinical Sciences Building, Edmonton, AB, Kanada; ${ }^{C}$ Zentrum für interstitielle und seltene Lungenerkrankungen, Abteilung für Pneumologie und Beatmungsmedizin der Thoraxklinik des Universitätsklinikums Heidelberg, Deutsches Zentrum für Lungenforschung, Thoraxklinik Heidelberg, Heidelberg, Deutschland; dSensory Motor Adaptive Rehabilitation Technology (SMART) Network, University of Alberta, Edmonton, AB, Kanada

\section{Schlüsselwörter}

Idiopathische pulmonale Fibrose · Komorbiditäten · Alterung · Seneszenz · gastroösophagealer Reflux · koronare Herzkrankheit · Emphysem · Überlebensdauer

\begin{abstract}
Zusammenfassung
Die idiopathische Lungenfibrose (IPF) ist eine chronische progrediente Erkrankung mit einer geschätzten medianen Überlebensdauer von 3 bis 5 Jahren ab der Diagnose. Sie tritt vorwiegend bei Älteren auf, und epidemiologische Studien sprechen dafür, dass die Hauptrisikofaktoren, die Alterung und Exposition gegenüber Zigarettenrauch, sowohl mit pulmonalen als auch extrapulmonalen Komorbiditäten (definiert als Auftreten von zwei oder mehr Erkrankungen bei einer Person) assoziiert sind. Alterung und Seneszenz können durch Wechselwirkungen mit Umweltfaktoren über verschiedene Mechanismen an der Pathogenese der IPF beteiligt sein, da sie das Lungenepithels schädigen und die Apoptoseresistenz der Myofibroblasten erhöhen, wodurch es schließlich zu einer Akkumulation von extrazellulärer Matrix und zu einer Lungenfibrose kommt. Syndrome mit kurzen Telomeren, bei denen es sich um archetypische vorzeitige Alterungssyndrome handelt und die häu-
\end{abstract}

fig mit Lungenfibrose einhergehen, sind dafür ein Paradigma. Die pathophysiologischen Charakteristika, die durch Alterung und Seneszenz bei Patienten mit IPF verursacht werden, können sich in Form pulmonaler und extrapulmonaler Merkmale äußern, darunter Emphysem, pulmonale Hypertonie, Lungenkrebs, koronare Herzkrankheit, gastroösophagealer Reflux, Diabetes mellitus und etliche andere chronische Erkrankungen, die mit erheblichen negativen Auswirkungen auf verschiedene Outcome-Parameter bei IPF verbunden sein können. Daher bietet die sorgfältige Diagnosestellung und Behandlung von Komorbiditäten eine ausgezeichnete Möglichkeit, Lebensqualität und Überlebensdauer zu verbessern, und alle in Frage kommenden Optionen für das IPF-Management, einschließlich Früherkennung und Behandlung von Komorbiditäten, sind in Betracht zu ziehen.

(c) 2021 Luppi, Kalluri, Faverio, Kreuter, Ferrara

\section{Einleitung}

Die idiopathische Lungenfibrose (IPF) ist eine chronische progrediente Erkrankung mit einer geschätzten medianen Überlebensdauer von 3 bis 5 Jahren ab der Diagnose. Für die Diagnose einer IPF ist das typische Muster einer gewöhnlichen inter- stitiellen Pneumonie (usual interstitial pneumonia, UIP), nachgewiesen mittels hochauflösender Computertomographie (high-resolution computed tomography, HRCT) oder histologischer Untersuchung in einem entsprechenden klinischen Setting, und das Fehlen einer identifizierbaren Ätiologie erforderlich [1]. 
Kohortenstudien sprechen dafür, dass verschiedene IPF-Phänotypen existieren [2], bei denen manche Patienten lange Phasen der Stabilität erleben [3], wohingegen es bei anderen zu Exazerbationen $[4,5,6]$ oder einer schnellen progredienten Verschlechterung kommt [7].

Es wurde gezeigt, dass die beiden antifibrotischen Arzneimittel Pirfenidon und Nintedanib die Verschlechterung der Lungenfunktion bei Patienten mit leichter bis mittelschwerer Erkrankung verlangsamen $[8,9]$, doch bestehen unter Umständen noch andere Möglichkeiten der Intervention, um die Prognose von IPF-Patienten zu verbessern [10, 11].

Die IPF betrifft vorwiegend ältere Menschen, und epidemiologische Kennzeichen lassen vermuten, dass verschiedene Risikofaktoren, darunter Alterung und genetische Veränderungen, die die Anfälligkeit für die Erkrankung erhöhen, sowie Umweltfaktoren, vor allem die Exposition gegenüber Zigarettenrauch, der Pathogenese der Erkrankung und ihren Komorbiditäten zugrunde liegen könnten. Zwar gilt die IPF als eine auf die Lunge begrenzte Erkrankung, doch hat sie gemeinsame Risikofaktoren mit zahlreichen Komorbiditäten (wie beispielsweise kardiovaskulären und degenerativen Erkrankungen [12, 13]), die möglicherweise eine wichtige Rolle für den Krankheitsverlauf von IPF-Patienten spielen.

Definitionsgemäß kommt es bei der Alterung zu einer fortschreitenden Abnahme der physiologischen Funktionen, die zu einem Anstieg der altersspezifischen Mortalitätsrate führt [14]; dabei handelt es sich um einen komplexen Prozess, an dem mehrere Mechanismen auf verschiedenen Ebenen beteiligt sind. Derzeit geht man davon aus, dass im Lauf der Zeit zu einer Akkumulation von Zellschäden erfolgt, die grundsätzlich zufälliger Natur sind und die normalerweise durch genetische Erhaltungs- und Reparaturmechanismen ausgeglichen werden. Die zunehmende Akkumulation zellulärer Defekte hat Auswirkungen auf den gesamten Organismus und führt schließlich zu altersbedingter Gebrechlichkeit, Behinderung und Krankheit [15].

Die beiden Hauptrisikofaktoren für IPF, das heißt Alterung und Zigarettenrauch, sind mit häufigen Komorbiditäten - definiert als Auftreten von zwei oder mehr Erkrankungen bei einer einzelnen Person - assoziiert [16] (Tab. 1).

Erste Hinweise lassen vermuten, dass die Prävalenz und die klinischen Auswirkungen dieser Komorbiditäten wahrscheinlich zunehmen werden, wenn sich die Überlebensdauer bei IPF durch die derzeitigen und zukünftigen Interventionen verbessert. Allerdings sind die in pharmazeutischen Studien gewonnenen Daten nicht immer auf die allgemeine IPF-Population übertragbar, da der Ausschluss von Patienten mit signifikanten Komorbiditäten in den Kohorten pharmazeutischer Studien der wichtigste Selektionsbias ist [17].

Die vorliegende Übersichtsarbeit beleuchtet die Bedeutung des Alterns, seine Mechanismen sowie das Wechselspiel mit anderen Risikofaktoren, wie etwa Zigarettenrauch, bei der Feststellung der IPF und ihrer Komorbiditäten und zeigt auf, dass Erkrankungen, die direkt oder indirekt mit der Lungenfibrose zusammenhängen, einen ähnlichen Ursprung haben können und dass ihre Be-
Tab. 1. Häufige Komorbiditäten und Krankheiten bei IPF-Patienten

\begin{tabular}{|c|c|}
\hline Pulmonal & Extrapulmonal \\
\hline Pulmonale arterielle Hypertonie & Koronare Herzkrankheit \\
\hline Emphysem & Angstzustände und Depression \\
\hline Obstruktive Schlafapnoe & Dekonditionierung und Sarkopenie \\
\hline Lungenkrebs & Osteoporose und Knochenfrakturen \\
\hline Venöse Thromboembolie & Diabetes mellitus und Hypothyreose \\
\hline Chronisch obstruktive & Gastroösophageale Refluxkrankheit \\
\hline
\end{tabular}

handlung spezifische diagnostische Ansätze und kombinierte Interventionen erfordert.

\section{Molekulare Grundlagen der IPF: Wie Alterung und Zigarettenrauch zusammenwirken}

Altern ist ein komplexer Prozess, bei dem genomische Instabilität, Telomerverkürzung, epigenetische Veränderungen, Verlust der Proteostase, eine fehlregulierte Nährstoffsensorik, mitochondriale Funktionsstörungen, zelluläre Seneszenz, Stammzellenerschöpfung und Veränderungen der interzellulären Kommunikation eine Rolle spielen [15]. Insbesondere ist die zelluläre Seneszenz definiert als ein Zustand dauerhaften Wachstumsstopps in Kombination mit bestimmten phänotypischen Veränderungen, die Chromatin- und Sekretomveränderungen umfassen [18]. Verschiedene Stimuli, darunter Zigarettenrauch [19], können über umfassend beschriebene Signalnetzwerke, die auf Tumorsuppressor-Signalwegen zusammenlaufen und einen dauerhaften Zellzyklusarrest induzieren, Seneszenz vermitteln [20].

Die aberrante Aktivierung alveolärer Epithelzellen, die wahrscheinlich durch Umweltfaktoren wie Zigarettenrauch oder Luftverschmutzung bestimmt wird [21], sowie die Akkumulation von Fibroblasten und Myofibroblasten, die zu einer übermäßigen Produktion von extrazellulärer Matrix führt, werden derzeit als die charakteristischsten pathogenetischen IPF-Merkmale angesehen [22]. In diesem Szenario gilt die Alterung als wichtiger Risikofaktor für die Entwicklung einer IPF, auch wenn die Mechanismen, über die Alterung und Umweltfaktoren (wie Zigarettenrauch) bei der Entwicklung der Krankheit miteinander verbunden sind, zum Großteil noch unklar sind [23].

Eine Vielzahl von Zelltypen in der Lunge von IPF-Patienten unterliegt der Alterung und ihren Folgen [24, 25]. Die Alterung wirkt sich sowohl auf die angeborene als auch auf die adaptive Immunität aus und führt zu einer Beeinträchtigung der zellulären Abwehrmechanismen gegen Krankheitserreger und Umwelteinflüsse wie Zigarettenrauch [14]. Letztlich beruhen die Unterschiede bei den Gewebe-Umbauprozessen (Remodelling) bei IPF möglicherweise auf den spezifischen beteiligten Zelltypen oder ihrem sich daraus ergebenden Schicksal (Apoptose gegenüber Apoptoseresistenz) [14]. Zudem kann die Erschöpfung von Zellpopulationen (wie Stammzellen und Perizyten) in der Lunge zum Verlust des Gleichgewichts zwischen epithelialen und mesenchy- 


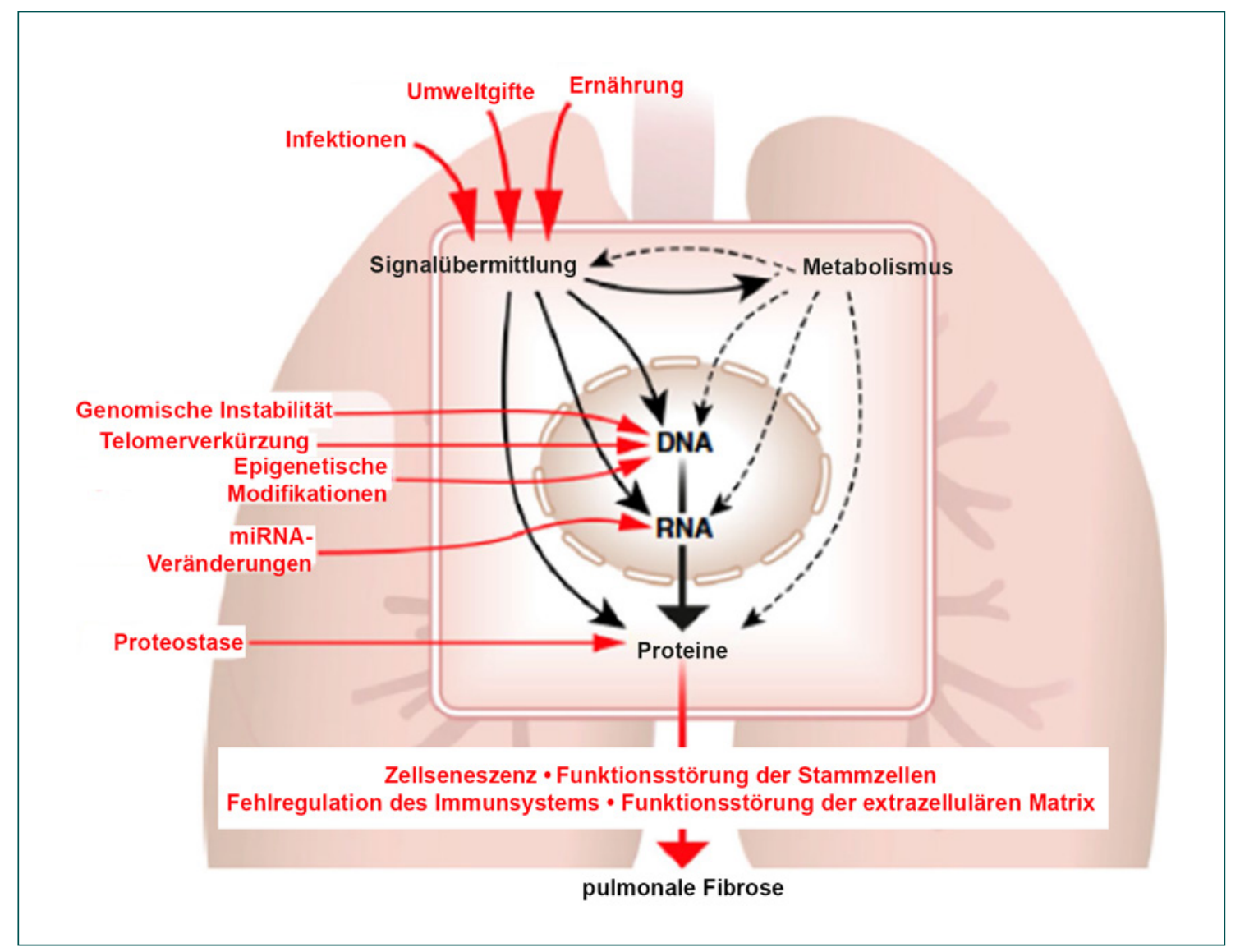

Abb. 1. Merkmale der Alterung auf molekularer Ebene, die zu Lungenfibrose führen; modifiziert nach [14].

malen Zellen beitragen, wodurch es zu einer Aktivierung und Proliferation von Fibroblasten kommt [26].

Die gegenwärtige Evidenzlage deutet darauf hin, dass die Hauptmerkmale der Alterung bei IPF vorzeitig auftreten und primär die Epithelzellen betreffen [22]. Konkret könnte der Tod der alveolären Epithelzellen zumindest teilweise für die gestörte Re-Epithelisierung sowie die Funktionsstörung des Epithels und den Verlust der Parenchymarchitektur verantwortlich sein [22]. Ferner kann die Alterung durch Sekretion zahlreicher verschiedener Mediatoren, die an der Entwicklung und Aufrechterhaltung der Fibrosierung beteiligt sind, tiefgreifende Auswirkungen auf die Mikroumgebung der Lunge haben [22]. Alveoläre Typ-II-Zellen in der Lunge von IPF-Patienten weisen zudem eine deutliche Akkumulation von dysmorphen und dysfunktionalen Mitochondrien sowie eine gestörte Autophagie auf, was ebenfalls zur abnormen Fibrosierung beitragen kann [27]. Die Abnahme der Mitochondrienfunktion und die verringerte Autophagie-Aktivität sind der gemeinsame Nenner altersbedingter Erkrankungen.

Vor Kurzem wurde gezeigt, dass neben Alveolarepithelzellen auch Fibroblasten/Myofibroblasten in Fibroblastenherden Marker der Zellseneszenz aufweisen [25]. Seneszente Fibroblasten sind überwiegend nicht proliferierend und zeigen wenig Anzeichen von Apoptose, was möglicherweise dazu beiträgt, dass sie vermindert eliminiert werden [28].
Zusammenfassend lässt sich sagen, dass Alterung und Seneszenz durch Wechselwirkungen mit Umweltfaktoren über zwei Mechanismen an der Pathogenese der IPF beteiligt sein können; diese führen zu einem abnormen Sekretionsmuster des Lungenepithels und erhöhen die Apoptoseresistenz der Myofibroblasten, wodurch es zu einer Akkumulation von extrazellulärer Matrix und Lungenfibrose kommt (Abb. 1).

\section{Beschleunigte Alterung: die Bedeutung der Telomere und anderer Genmutationen sowie ihre klinischen Auswirkungen}

Die Länge der Telomere nimmt physiologischerweise mit zunehmendem Alter ab und ist ein Prädiktor für das Einsetzen der replikativen Seneszenz. Ein wichtiges Enzym, das die Chromosomenenden wiederherstellt, ist die Telomerase [29]. Die familiäre interstitielle Pneumonie tritt gewöhnlich in einem jüngeren Alter auf als die nicht familiäre IPF [30]. Darüber hinaus sind Mutationen in den essenziellen Genen, die für das Enzym Telomerase kodieren, die am häufigsten identifizierten Mutationen bei IPF [31]. Die Telomerase besteht aus zwei essenziellen Komponenten: der Telomerase-Reverse-Transkriptase (hTERT) und der hTR, eine spezialisierte RNA, die eine Matrize für die Addition von Telomer-Repeats enthält. Mindestens einer der möglichen Grün- 
de für den Unterschied im Alter bei Krankheitsbeginn zwischen familiärer und sporadisch auftretender Lungenfibrose erklärt sich aus der kürzlich erfolgten Beschreibung von Keimbahnmutationen in den Genen hTERT und hTR, die mit dem TelomeraseKomplex assoziiert sind [32]. Mutationen in den Telomerase- und Telomergenen kennzeichnen die Dyskeratosis congenita, ein seltenes Syndrom, bei dem es zu einer vorzeitigen Alterung kommt und das vor einem Jahrhundert entdeckt wurde [33]. Ungefähr einer von fünf Patienten mit Dyskeratosis congenita entwickelt schließlich eine Lungenfibrose, und bei etwa 15\% der Patienten mit familiärer Lungenfibrose finden sich Telomerase-Mutationen [34]. In einer Reihe von Telomer-bezogenen Genen, darunter TINIF2, Nuclear Assembly Factor 1 (NAF1), Dyskerin-Pseudouridin-Synthase 1 (DKC1) und Regulator of Telomere Elongation Helicase 1 (RTEL1), wurden Mutationen identifiziert und mit IPF und anderen interstitiellen Lungenerkrankungen (interstitial lung diseases, ILDs) in Verbindung gebracht [35]. Mehr als zwei Drittel der betroffenen Patienten mit diesen Mutationen haben eine Rauchervorgeschichte [36]. Tatsächlich sind die Telomere, auch im Alveolarepithel [38], bei aktuellen und ehemaligen Rauchern kürzer als bei den nach Alter vergleichbaren Kontrollen [37], und die Telomerase-Aktivität wird durch Sexualhormone gesteuert [39], was möglicherweise dazu beiträgt, dass die Lungenfibrose bei Männern häufiger auftritt.

Dem derzeitigen Konzept zufolge sind Mutationen in Telomerase- und Telomerkomponenten bei Erwachsenen ein prädisponierender Faktor für eine Vielzahl von Erkrankungen, die durch Lungenfibrose, Leberfibrose und hämatologische Charakteristika gekennzeichnet sind [40], wobei das Alter bei Krankheitsbeginn und der Schweregrad durch die Telomerlänge bestimmt werden. Zwar sind die genauen pathophysiologischen Mechanismen nicht bekannt, doch könnte der Verlust der Telomerase-Aktivität durch Unterdrückung der Differenzierung von Fibroblasten zu Myofibroblasten [41] und durch die Seneszenz der Alveolarepithelzellen, die die alveoläre Reparatur einschränkt, an der Entwicklung einer Lungenfibrose beteiligt sein [42]. Insgesamt ist zu sagen, dass es sich bei den Syndromen mit kurzen Telomeren um archetypische Syndrome der vorzeitigen Alterung handelt, die häufig mit Lungenfibrose assoziiert sind.

Neben anderen Genen, die möglicherweise mit der Zellseneszenz in Zusammenhang stehen, war die Variante rs35705950 in der Promotorregion des Mucin 5B (MUC5B)-Gens in einer genomweiten Linkage-Analyse mit einem etwa siebenfach erhöhten IPFRisiko verbunden [43]. Diese MUC5B-Variante wurde in verschiedenen Studien validiert und gilt nach wie vor als Hauptrisikofaktor für IPF [44].

Mittels genomweiter Assoziationstechnologie wurden drei häufige, mit IPF assoziierte Varianten (rs111521887, rs5743894, rs574389) im Toll-interacting protein (TOLLIP)-Gen ermittelt, von denen eine (rs5743894) mit einem verringerten IPF-Risiko, jedoch einer erhöhten Mortalität der an IPF Erkrankten assoziiert war [45].

In einer kürzlich von Fingerlin et al. durchgeführten genomweiten Assoziationsstudie zu IPF und fibrosierender idiopathischer interstitieller Pneumonie (IIP) wurden insgesamt 2492 Patienten mit fibrosierender IIP (die meisten davon IPF) erfasst und mit mehr als 6000 Kontrollprobanden verglichen. In dieser Studie wurden sieben neue Loci ermittelt, die mit Genen in Zusammenhang stehen, welche die Wirtsabwehr, die Zell-Zell-Adhäsion und die DNA-Reparatur steuern und die zum Risiko, eine Fibrose zu entwickeln beitragen [46] und die daher mit der Zellseneszenz verbunden sind.

\section{Die Bedeutung von Komorbiditäten und Krankheiten im Zusammenhang mit IPF}

Eine aktuelle systematische Übersichtsarbeit bestätigte eine hohe Prävalenz vieler Komorbiditäten bei IPF-Patienten im Vergleich zur Allgemeinbevölkerung [47]. Darüber hinaus wiesen nach Berichten einer kürzlich veröffentlichte Studie etwa $60 \%$ der IPFPatienten eine bis drei Komorbiditäten auf, 30\% hatten vier bis sieben Komorbiditäten und nur 10\% hatten keine Komorbiditäten. Dies verdeutlicht, dass die kumulative Inzidenz der Komorbiditäten klinisch relevant ist und die Überlebensdauer beeinflusst [48] (Abb. 2). Die Prävalenz von Komorbiditäten bei IPFPatienten fällt je nach untersuchtem Patientenkollektiv und diagnostischen Kriterien sehr unterschiedlich aus. Die Komorbiditäten haben jedoch zusätzliche physiologische und symptomatische Auswirkungen und können daher vor dem Hintergrund der zugrunde liegenden Lungenfibrose einen signifikanten Einfluss auf die IPF haben, und möglicherweise sind sie zudem auch für andere IPF-Phänotypen charakteristisch.

\section{Koronare Herzkrankheit}

Neueren Studien zufolge kommt die koronare Herzkrankheit (KHK) bei Lungentransplantationskandidaten mit IPF signifikant häufiger vor als bei denjenigen mit Emphysem (etwa 10\% gegenüber 30\%) [49]. Ebenso wurde gezeigt, dass fibrosierende Lungenerkrankungen nach Adjustierung für die üblichen Risikofaktoren mit einer höheren KHK-Prävalenz assoziiert waren als nicht fibrosierende Erkrankungen [50]. Nathan et al. bestätigten die Assoziation von IPF und KHK ebenfalls, indem sie IPF-Patienten mit einer Kontrollkohorte von Patienten mit chronisch-obstruktiver Lungenerkrankung (chronic obstructive pulmonary disease, COPD) verglichen und nachwiesen, dass IPF-Patienten häufiger eine schwere KHK hatten als COPD-Patienten und diese mit einer erhöhten Mortalität verbunden war [51].

Die hochauflösende Computertomographie des Thorax wird für alle Patienten mit (Verdacht auf) IPF als obligatorisches Instrument für die Diagnose der IPF empfohlen und kann daher auch ein geeignetes Screening-Instrument für KHK bei Hochrisikopopulationen darstellen. Der Nachweis von mittelschweren bis schweren koronararteriellen Verkalkungen in der HRCT besaß eine hohe Sensitivität und Spezifität für die Diagnose einer KHK [52]. Dieser radiologische Befund sollte Kliniker dazu bringen, bei IPF-Patienten mit mäßigen bis schweren Koronarkalzifizierungen in der HRCT-Aufnahme eine Überweisung zum Kardiologen zu erwägen. 


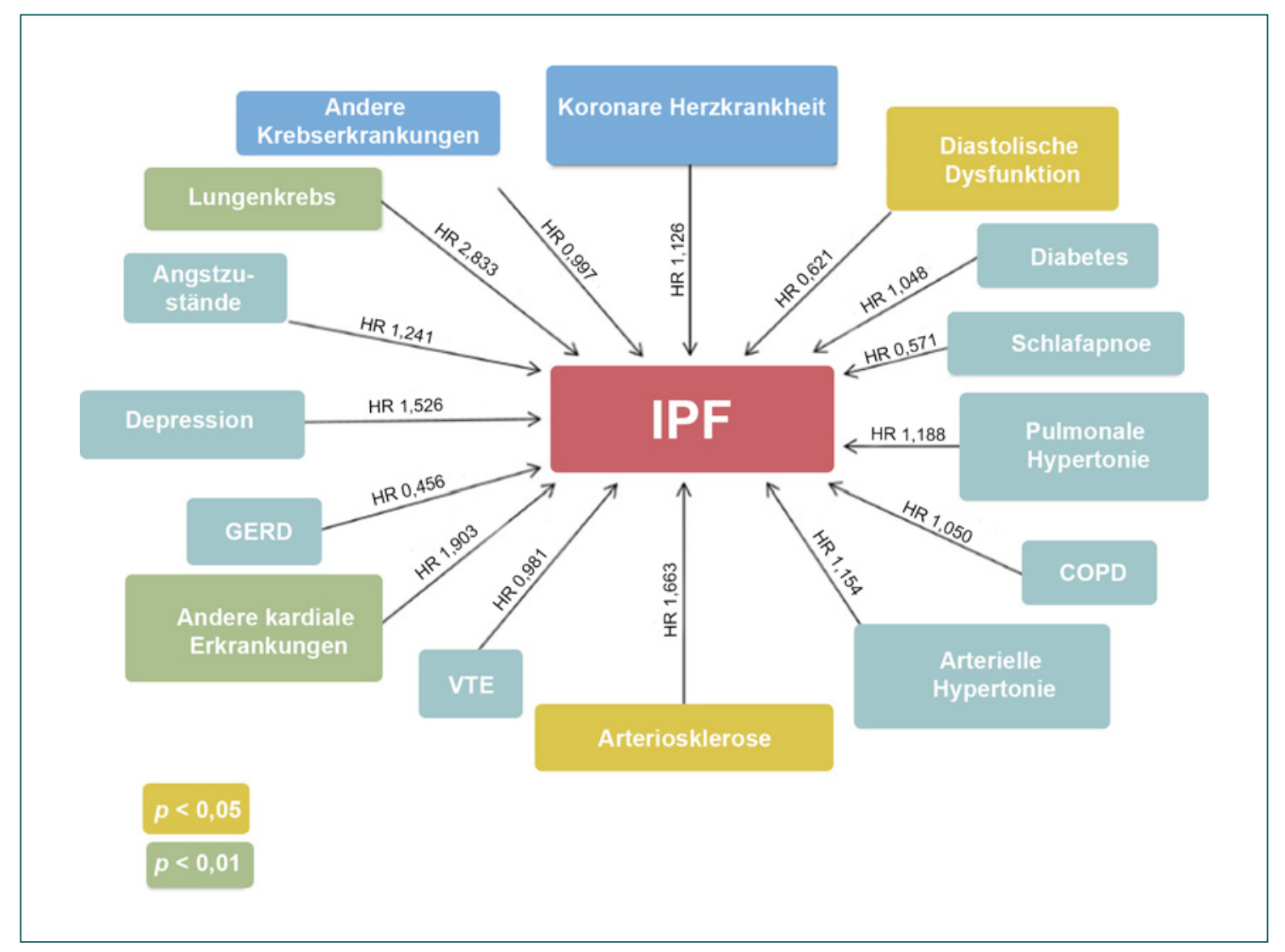

Abb. 2. Auswirkungen der idiopathischen pulmonalen Fibrose und Komorbiditäten auf die Mortalität; modifiziert nach [41].

Die hohe Prävalenz dieser Komorbidität bei IPF-Patienten lässt sich zum Teil durch eine gemeinsame genetische Prädisposition für die Entwicklung einer IPF und KHK erklären, sowie durch den Nachweis spezifischer Interleukin (IL)-17-Immunreaktionen auf die Alpha-1-Kette von Kollagen Typ V und durch ihre Korrelation mit humanen Leukozytenantigen (HLA)-D15-Allelen [53] Allerdings spielen die Rauchervorgeschichte und die erhöhte Prävalenz von Diabetes mellitus bei diesen Patienten, wie im Folgenden erörtert, möglicherweise ebenfalls eine Rolle.

\section{Andere kardiale Komorbiditäten}

Belege mit niedriger Evidenzstärke deuten darauf hin, dass auch andere kardiale Komorbiditäten, einschließlich Arrhythmien wie Vorhofflimmern und Herzinsuffizienz oder kongestive Herzinsuffizienz, häufig bei Patienten mit IPF auftreten können [54]. Daten aus Tiermodellen sprechen dafür, dass bei den kardialen und pulmonalen Remodelling-Prozessen bei Herzinsuffizienz der Bindegewebe-Wachstumsfaktor (connective tissue growth factor, CTGF) eine Rolle spielen könnte [55, 56]. Es ist bekannt, dass in den Fibroblasten von IPF-Patienten eine Überexpression von CTGF vorliegt [57]. Zukünftige Studien werden klären, ob dieser Wachstumsfaktor möglicherweise das Auftreten von kardiovaskulären Komorbiditäten während des natürlichen Verlaufs der IPF beeinflusst.

\section{Hämatologische Anomalien}

Die IPF geht mit einem prothrombotischen Zustand einher, der für die Pathogenese von Bedeutung sein kann [58]. Entsprechend deuteten epidemiologische Studien zudem auf eine starke Assoziation zwischen IPF und venösen Thromboembolien (VTE) [59] und dadurch bedingten Lungenembolien [60] hin, die daher als Komorbidität der IPF angesehen werden können. Dennoch scheint es nach den Ergebnissen randomisierter kontrollierter Studien (randomized controlled trials, RCTs), in denen die Wirksamkeit von Antikoagulantien bei IPF-Patienten untersucht wurde, dass diese einen nachteiligen Effekt auf die Überlebensdauer haben $[61,62,63]$; daher werden sie in der Behandlung der IPF nicht empfohlen [64].

Mutationen in den hTERT- und hTR-Genen sind ebenfalls mit hämatologischen Anomalien, wie beispielsweise aplastische Anämie, Makrozytose, Thrombozytopenie und Myelodysplasie/ Leukämie verbunden [65, 66]. Das Risiko einer Myelotoxizität bzw. eines Knochenmarkversagen nach Lungentransplantation ist bei IPF höher als bei anderen Patienten und sollte bei der Beurteilung von Patienten für eine Transplantation sorgfältig bewertet werden [67]. 


\section{Osteoporose und Knochenfrakturen}

In einige Studien wurde der Zusammenhang zwischen IPF und Knochenstatus untersucht und gezeigt, dass bei Patienten, die keine Kortikosteroidbehandlung erhielten, eine reduzierte Knochenmineraldichte der Brustwirbel zu beobachten war [68]. Eine kürzlich durchgeführte populationsbasierte Studie ergab, dass etwa ein Drittel der IPF-Patienten Osteoporose und mindestens eine Wirbelfraktur hat. Eine verringerte Knochenmineraldichte war mit einer signifikanten Abnahme der FVC und der Kohlenmonoxid-Diffusionskapazität (diffusing capacity of the lungs for carbon monoxide, DLCO) assoziiert [69].

Die Platelet-derived growth factor (PDGF) Untereinheit B wurde sowohl mit Osteoporose [70] als auch mit fibrosierender ILD [71] in Zusammenhang gebracht, doch ist ihre Rolle in der Pathogenese dieser Erkrankungen noch nicht vollständig geklärt.

Vitamin D steht ebenfalls mit dem Knochenstoffwechsel in Verbindung. In einer vor Kurzem veröffentlichten Studie wiesen IPFPatienten niedrige Vitamin-D-Serumspiegel auf, und ein solcher Mangel korrelierte mit der Gesamtmortalität, was auf seine mögliche Rolle als prognostischer Faktor und therapeutischer Angriffspunkt hindeutet [72].

\section{Diabetes mellitus}

Verschiedene Studien ergaben, dass die Prävalenz von Diabetes mellitus bei IPF-Patienten [73, 74, 75] im Vergleich zur Allgemeinbevölkerung erhöht ist. In einer dieser Studien wurde die stärkste Assoziation bei Anwendung von Insulin beobachtet, und die höhere Prävalenz bei IPF-Patienten blieb auch dann bestehen, nachdem Patienten mit Kortikosteroidtherapie ausgeschlossen worden waren [74]. Die an dieser Assoziation beteiligten pathogenetischen Mechanismen sind unklar. Möglicherweise bewirken hohe Glukosespiegel eine Aktivierung verschiedener Signalwege, die mit der Bildung reaktiver Sauerstoffspezies und profibrotischer Zytokine in Zusammenhang stehen [3], oder unter Umständen, ähnlich wie bei Mukoviszidose, mit einer gestörten bakteriellen Clearance bei hyperglykämischen Zuständen.

Interessanterweise hat sich gezeigt, dass eine abnorme Telomerverkürzung mit Diabetes mellitus Typ 2, Insulinresistenz und gestörter Glukosetoleranz assoziiert ist [76].

Advanced Glycation Endproducts (AGEs) werden als entscheidender Faktor für arterielle und vaskuläre Schädigungen und die Entzündung bei Diabetes angesehen [77]. RAGE-Polymorphismen im RAGE (Receptor for advanced glycation end products)Signalweg wurden mit Demenz, einem erhöhten Krebsrisiko sowie einem erhöhten Risiko für kardiovaskuläre Erkrankungen in Verbindung gebracht [78]. Der lösliche RAGE-Ligand (soluble RAGE ligand, sRAGE) steuert der RAGE-Aktivierung durch Advanced Glycation Endproducts entgegen.

Zudem scheint die nukleäre RAGE-Isoform (nRAGE) wesentlich für die Erhaltung der Reparatur von DNA-Doppelstrangbrüchen zu sein, und die Modulation dieses nukleären Mediators gekoppelt mit einer ATM-Kinase hat das Potenzial, bei Mäusen die Fibrose umzukehren [79]. Der Zusammenhang zwischen dem RAGE-Signalweg und der Pathogenese von Diabetes mellitus und IPF ist noch unerforscht. Die erste Studie zur möglichen Rolle des RAGESignalwegs bei IPF zeigte jedoch, dass die Konzentration an zirkulierendem sRAGE bei IPF-Patienten verringert war, insbesondere wenn sie das rs2070600 A-Allel trugen, und dass eine Korrelation mit dem Schweregrad der Erkrankung bestand [80].

\section{Hypothyreose}

Oldham et al. wiesen in einer retrospektiven Fall-Kontroll-Analyse nach, dass Hypothyreose bei IPF-Patienten häufig und mit einer deutlich höheren Prävalenz (13\% der betroffenen Männer und 28\% der betroffenen Frauen) auftritt als in der Allgemeinbevölkerung (1-2\% der Männer und 5-9\% der Frauen) [81]. Die potenzielle Bedeutung dieser Komorbidität wird dadurch unterstrichen, dass Hypothyreose prädiktiv für die Mortalität bei IPF ist [81].

Die Beziehung zwischen der Schilddrüse und der Lunge ist komplex, und die Mechanismen sind in der Tiefe nach wie vor unbekannt. Mutationen des thyroidalen Transkriptionsfaktors NK2 homeobox 1 (NKXS2-1) sind mit dem Hirn-Lunge-Schilddrüsen-Syndrom assoziiert, das Neugeborene und Kinder mit histologischen Merkmalen einer Surfactant-Störung betrifft [82]. Das Vorliegen einer Autoimmunhypothyreose ist darüber hinaus mit einer erhöhten Mortalität bei chronischer Hypersensitivitätspneumonie assoziiert [83]. Auch wurden anekdotische Fälle einer Umkehrung der Lungenfibrose in vivo bei Einleitung einer Thyroxin-Substitutionstherapie beschrieben; diese sind bisher allerdings nur unzureichend charakterisiert [84].

\section{Emphysem}

Die kombinierte pulmonale Fibrose und Emphysem (combined pulmonary fibrosis and emphysema, CPFE) ist definiert als gleichzeitiges Vorliegen eines Oberlappenemphysems und einer basalen Fibrose. Der Begriff wurde 2005 von Cottin et al. geprägt [85], obschon bereits früher über diese Koexistenz berichtet worden war [86]. Ob die CPFE eine eigenständige Krankheitsentität darstellt oder die Koinzidenz von zwei pulmonalen Erkrankungen im Zusammenhang mit Zigarettenrauchen beschreibt, ist bislang nicht geklärt. Die berichtete Prävalenzspanne der CPFE ist breit und beträgt zwischen 8 und 51\%, wobei jedoch der Anteil an IPF-Patienten im Median bei etwa einem Drittel liegt [87] und die Möglichkeit besteht, einen spezifischen Phänotyp von IPF-Patienten zu identifizieren, die eine CPFE entwickeln können; in der Regel ältere Männer und ausnahmslos aktuelle oder ehemalige Raucher, die häufig eine ausgeprägte Entsättigung unter Belastung zeigen [87]. Die Diagnose dieser Erkrankung wird mittels HRCT des Thorax gestellt. Oftmals haben die die Patienten eine ausgeprägte Entsättigung unter Belastung, und Lungenfunktionstests zeigen häufig ein erhaltenes Lungenvolumen mit einer stark verringerten Kohlenmonoxid-Diffusionskapazität [88]. Die Kombination aus entgegengesetzten obstruktiven und restrikti-
236

Kompass Pneumol 2021;9:230-242 DOI: $10.1159 / 000518426$ 
ven Volumina bestimmt ein erhaltenes Lungenvolumen mit stark verminderter Diffusionskapazität. Pulmonale Hypertonie (PH) ist eine weitere häufige Komorbidität $[85,89]$ und mit einer negativen Prognose verbunden [90]. Darüber hinaus ist die CPFE jedoch auch mit einem erhöhten Risiko für andere Komorbiditäten, einschließlich Lungenkrebs, assoziiert. In der Tat ist die Prävalenz von Lungenkrebs bei diesen Patienten höher als bei Patienten mit Emphysem oder alleiniger IPF [87]. Patienten mit CPFE und Lungenkrebs haben eine signifikant schlechtere Überlebensdauer als Patienten mit Emphysem und Lungenkrebs allein, was dafür spricht, dass die «Triple-Hit»-Effekte von Rauchen, Emphysem und Lungenfibrose die Prognose von CPFE-Patienten verschlechtern können [91]. Eine weitere klinisch relevante Komorbidität der CPFE ist das Auftreten akuter Exazerbationen, die denen bei IPF-Patienten ähnlich sind. Verschiedene Risikofaktoren können bei CPFE-Patienten zu akuten Exazerbationen führen, darunter auch eine Lungenresektion [87] in Verbindung mit Strahlen- und Chemotherapie bei Patienten mit gleichzeitig vorliegendem Lungenkrebs.

Bemerkenswert ist, dass die Telomerlänge und Mutationen im TERT/TERC-System sowohl mit der Emphysemanfälligkeit [92] als auch mit CPFE $[93,94]$ in Zusammenhang gebracht wurden, und dies stützt das Konzept, nach dem beide Manifestationsformen Teil eines Alterungsprozesses der Lunge sind, der durch genetische und Umweltfaktoren bestimmt wird.

Da bislang keine klinische Studie durchgeführt wurde, in der speziell CPFE-Patienten untersucht wurden, basieren die Behandlungsempfehlungen bei diesen Patienten auf Expertenmeinungen. Gegebenenfalls sollten die Aufgabe des Rauchens, Impfungen, Sauerstoffgabe und eine pulmonale Rehabilitation verordnet werden. Der Nutzen einer Behandlung von CPFE-Patienten mit inhalativen langwirksamen Anticholinergika, inhalativen langwirksamen Beta-Agonisten und/oder inhalativen Kortikosteroiden ist unklar [95]. Patienten ohne Kontraindikationen sollten gegebenenfalls zur Beurteilung einer Lungentransplantation an eine entsprechende Klinik überwiesen werden.

\section{Angstzustände und Depression}

Da die IPF eine chronische progrediente Erkrankung ist, die erhebliche Auswirkungen auf die Morbidität und Mortalität hat, geht sie häufig mit Angst und Depression einher. Typische Merkmale der IPF-Progression sind insbesondere eine Verschlimmerung der Dyspnoe, die Verschlechterung der Lungenfunktion und Hypoxämie, die nachgewiesenermaßen mit Depression und Angstzuständen korrelieren [96], und in Verbindung mit Husten und Dyspnoe wichtige Determinanten der gesundheitsbezogenen Lebensqualität [96] und häufige Komorbiditäten bei IPF-Patienten sind. Die Prävalenz von Angstzuständen und Depressionen ist hoch (31\% bzw. 23\%) [96], weshalb alle Patienten, bei denen eine IPF diagnostiziert wird und die sich deswegen unter Nachbeobachtung befinden, auf diese Erkrankungen untersucht werden sollten [87]. Als Folge der Unterschätzung und Unterdiagnose wurde nur in einem Viertel der Fälle eine medikamentöse Thera- pie verschrieben, was darauf schließen lässt, dass ein Großteil der Patienten trotz der hohen Prävalenz nicht behandelt wird [97]. Um die Lebensqualität von Patienten mit IPF zu verbessern, können eine kognitive Verhaltenstherapie und Medikamente gegen Depression empfohlen werden, allerdings wurde die Wirksamkeit dieser Behandlungen bei IPF-Patienten nicht validiert [87]. Es hat sich gezeigt, dass die pulmonale Rehabilitation zu einer anhaltenden Besserung der depressiven Symptomatik führt und sie sollte allen IPF-Patienten empfohlen werden, die eine Depression haben und Funktionseinschränkungen aufweisen [98].

Bei fortgeschrittener fibrosierender Lungenerkrankung wirkte sich eine palliativmedizinische Intervention positiv auf die Angstzustände und die Depression aus, was die Bedeutung der bestmöglichen supportiven Therapie bei diesen Patienten untermauert [11].

\section{Pulmonale Hypertonie (PH)}

Als pulmonale Hypertonie gilt definitionsgemäß ein durch Rechtsherzkatheteruntersuchung gemessener mittlerer pulmonalarterieller Druck (mean pulmonary artery pressure, mPAP) in Ruhe von 20 mmHg oder höher [99]. Der normale mPAP-Wert ( \pm Standardabweichung) beträgt $14 \pm 3 \mathrm{mmHg}$. Eine IPF-bedingte PH ist eine häufige Komorbidität bei dieser Erkrankung, vor allem im fortgeschrittenen Stadium, und gilt als Marker für eine schlechte Prognose [100, 101].

Eine Studie mit mehr als 6500 Patienten ergab jedoch, dass schon eine leichte pulmonale Hypertonie das Mortalitätsrisiko bei Patienten mit IPF erhöht [102]. Einige Studien sprechen zudem dafür, dass die präkapilläre PH bei IPF bei etwa 8 bis 15\% der Patienten bei der Erstuntersuchung vorliegt $[103,104]$, und dieser Anteil steigt bei IPF-Patienten im fortgeschrittenen und Endstadium der Erkrankung, bei denen die PH ein häufiges Krankheitsmerkmal $(>50-60 \%)$ ist $[105,106,107]$.

Bei manchen Komorbiditäten, wie obstruktiver Schlafapnoe, Thromboembolien oder kardialer diastolischer Dysfunktion, steigt die Häufigkeit der PH noch weiter an [87].

Generell ist die PH bei IPF-Patienten von leichtem bis mittlerem Schweregrad. Bei einigen Patienten (etwa 10\%) fällt der pulmonalarterielle Druck gemäß Lungenfunktionstests [108] oder HRCT-Fibrose-Score [109] jedoch im Verhältnis zur Schwere der Lungenerkrankung unverhältnismäßig aus. In der Vergangenheit wurde die Diskrepanz zwischen dem Grad der PH und dem Schweregrad der Lungenerkrankung als «überproportionale» $\mathrm{PH}$ bezeichnet. Heutzutage wurde dieser Begriff fallen gelassen, seitdem in den Leitlinien der Begriff der schweren pulmonalen Hypertonie für Patienten mit mPAP-Werten $\geq 35 \mathrm{mmHg}$ oder mPAPWerten $\geq 25 \mathrm{mmHg}$ bei Vorliegen eines niedrigen Herzzeitvolumens (Herzindex $<2,5 \mathrm{l} / \mathrm{min}$ ) eingeführt wurde [110].

Die Symptome der PH sind bei IPF-Patienten unspezifisch, und das Vorliegen von PH kann leicht übersehen oder die Diagnose verspätet gestellt werden. Konkret kann das Auftreten einer PH bei IPF durch vermehrte Dyspnoe, einen verringerten Gasaustausch in Ruhe, eine deutlich erniedrigte DLCO, schnelle Entsät- 
tigung bei Belastung, hohe BNP (Brain Natriuretic Peptide)-Spiegel, Rechtsherzdilatation in der Thorax-Röntgenaufnahme, beeinträchtigte Lebensqualität, geringere Belastungstoleranz und einen höheren Bedarf an zusätzlichem Sauerstoff gekennzeichnet sein [107]. Bei IPF-Patienten im Spätstadium war eine schnelle Progression der PH zu beobachten [106].

Zwar gilt die Rechtsherzkatheteruntersuchung (RHC) als Goldstandard für die Diagnose einer $\mathrm{PH}$, doch erfolgt das initiale Screening auf pulmonale Hypertonie in der Regel durch die transthorakale Echokardiographie, auch wenn deren Genauigkeit bei chronischen Lungenerkrankungen, einschließlich IPF, nach wie vor strittig ist [111].

Zur Prävention und Therapie der PH sind wegen der Hypoxie zusätzliche Sauerstoffgaben indiziert, obgleich keine Daten vorliegen, die den günstigen Effekt von Sauerstoff auf die Überlebensdauer dieser Patientengruppe stützen.

Es existieren keine zugelassenen zielgerichteten Therapien für $\mathrm{PH}$ bei IPF [112], und kürzlich wurden einige klinische Studien zu Vasodilatatoren mit negativem Ergebnis durchgeführt. Insbesondere zeigten Vasodilatatoren bei IPF mit oder ohne PH keine Wirksamkeit in Hinblick auf eine Verlangsamung der IPF-Progression $[113,114,115]$ und sie bewirkten keine Veränderung der kardiovaskulären Hämodynamik bei IPF-Patienten mit gleichzeitig bestehender PH [116, 117]. Eine Studie, in der IPF-Patienten mit nachgewiesener $\mathrm{PH}$ gemäß Rechtsherzkatheteruntersuchung Ambrisentan erhielten, wurde gestoppt nachdem eine andere Studie mit Ambrisentan keinen Vorteil in der Subgruppe der IPFPatienten mit bekannter PH ergeben hatte [114]. Eine weitere Studie zu Riociguat, einem löslichen Guanylatzyklase-Stimulator, wurde ebenfalls gestoppt, nachdem sich in einer Zwischenanalyse gezeigt hatte, dass Patienten im Interventionsarm ein höheres Risiko für Tod und andere schwerwiegende unerwünschte Ereignisse hatten als Patienten im Kontrollarm [118].

Der Phosphodiesterase-5-Inhibitor Sildenafil wurde bei Patienten mit fortgeschrittener IPF untersucht. In der groß angelegten placebokontrollierten Doppelblindstudie «STEP-IPF» wurden IPF-Patienten randomisiert einer Behandlung mit oralem Sildenafil oder Placebo zugewiesen. Primäres Zielkriterium war der Nachweis einer mindestens 20\%igen Verbesserung beim 6-Minuten-Gehtest (6MWT) nach 12 Wochen: Es wurde keine statistische Signifikanz erreicht, da bei lediglich 10\% der Patienten im Sildenafil-Arm gegenüber 7\% im Placebo-Arm eine Verbesserung zur beobachten war $(p=0,39)$. Allerdings fanden sich kleine, jedoch klinisch relevante Unterschiede bei den sekundären Zielkriterien arterielle Oxygenierung, DLCO, Grad der Dyspnoe und Lebensqualität bei Patienten, die Sildenafil erhielten [119]. Eine Post-hoc-Subgruppenanalyse von Patienten mit nachgewiesener PH ergab, dass die Sildenafil-Therapie auch die Gehstrecke verbesserte [120]. Zudem verdeutlichten die Ergebnisse einer kürzlich durchgeführten Netzwerk-Metaanalyse mögliche wichtige Unterschiede bei der Mortalität und schwerwiegenden unerwünschten Ereignissen zwischen verschiedenen therapeutischen Interventionen bei IPF. Diese Ergebnisse sprechen für einen potenziellen Vorteil von Nintedanib, Pirfenidon und Sildenafil in
Hinblick auf die Mortalität im Vergleich zu anderen Behandlungen [121]. Auf Grundlage dieser Daten sprechen sich die neuesten evidenzbasierten Leitlinien bedingt gegen die routinemäßige Anwendung von Sildenafil bei Patienten mit IPF aus [1], und es wurden klinische Studien veröffentlicht, in denen Sildenafil in Kombination mit einer antifibrotischen Therapie - sowohl Pirfenidon als auch Nintedanib - bei Patienten mit IPF-assoziierter PH untersucht wurde [122, 123, 124].

Eine kürzlich durchgeführte Phase-2b/3-Studie mit gepulsten Inhalationen von Stickstoffmonoxid bei Patienten mit fibrosierender ILD und komplizierender PH lieferte vielversprechende erste Ergebnisse und zeigte einen positiven Effekt dieser Behandlung auf die körperliche Aktivität bei insgesamt gutem Sicherheitsprofil und guter Verträglichkeit [120].

\section{Gastroösophageale Refluxkrankheit}

Bei gastroösophagealem Reflux (gastro-esophageal reflux, GER) kommt es zu einem Reflux von Mageninhalt in den Ösophagus, wobei ein gewisser Grad an GER physiologisch ist. Der gastroösophageale Reflux kann mit ösophagealen $\mathrm{pH}$-Werten von $<4,0$ bzw. $>4,0$ sauer oder nicht sauer sein. Eine gastroösophageale Refluxkrankheit (gastro-esophageal reflux disease, GERD) entsteht, wenn der Reflux von Mageninhalt Symptome oder Komorbiditäten verursacht [125].

Wichtig ist, dass GERD nicht gleichbedeutend mit GER oder einfachem Sodbrennen ist und dass das GERD-Syndrom definitionsgemäß mit negativen Folgeerscheinungen des Refluxes verbunden ist [65].

Die gastroösophageale Refluxkrankheit tritt bei Patienten mit IPF häufig auf $[126,127]$ und mit einer Prävalenz zwischen 70 und $90 \%$ ist sie häufiger zu beobachten als in der Allgemeinbevölkerung [128] oder bei Patienten mit anderen chronischen Lungenerkrankungen.

Ghebre und Raghu [129] stellten vor Kurzem die Hypothese auf, nach der die Ursache-Wirkungs-Beziehung zwischen GERD und IPF durch zwei verschiedene Mechanismen bestimmt wird: 1 . eine chronische Mikroaspiration verursacht rezidivierende Schädigungen des bronchiolären und alveolären Epithels und fördert daher bei anfälligen Personen die Fibrogenese, wodurch es zu einer IPF kommt; 2. die verminderte Compliance der fibrotischen Lunge bewirkt einen Anstieg des intrathorakalen Drucks, der zu einer Funktionsstörung des unteren Ösophagussphinkters, GERD und Mikroaspiration führt, was den Krankheitsprozess der IPF aufrecht erhält und/oder beschleunigt.

Die typischen GERD-Symptome, einschließlich Sodbrennen, treten nur bei 25 bis $65 \%$ der IPF-Patienten mit bestätigter GERDDiagnose auf; infolgedessen schließt das Fehlen von Symptomen die Diagnose GERD bei IPF-Patienten nicht aus [130].

Zwar können die 24-Stunden-pH-Metrie und Ösophagusmanometrie bei der Feststellung einer GERD hilfreich sein, doch ist kein bestes diagnostisches Flussdiagramm zur Diagnose einer GERD bei IPF-Patienten definiert und sollte daher personalisiert erfolgen. Hauptsächlich aus diesem Grund wird die Behandlung
238

Kompass Pneumol 2021;9:230-242

DOI: $10.1159 / 000518426$ 
einer GERD oftmals auf Grundlage der GER-Symptome begonnen [87].

Die gastroösophageale Refluxkrankheit scheint zudem ein Risikofaktor für akute Exazerbationen der IPF (AE-IPF) zu sein: Annahmen zufolge ist Mikroaspiration einer der Mechanismen, die AE-IPF auslösen, wie von Lee et al. nachgewiesen, die einen hohen Pepsinspiegel in der bronchoalveolären Lavageflüssigkeit von AE-IPF-Patienten feststellten. Der Nachweis von Pepsin in der bronchoalveolären Lavageflüssigkeit dieser Patienten wurde als Biomarker für eine Mikroaspiration vorgeschlagen und er korrelierte mit AE-IPF, wenngleich der erhöhte Pepsinspiegel kein Prädiktor für die Mortalität war [131].

Der Nutzen einer Antazida-Gabe in Hinblick auf die IPF-Progression wird nach wie vor kontrovers diskutiert. Fallserien und unkontrollierte Studien zeigten günstige Effekte der GERD-Therapie bei IPF, darunter auch eine Stabilisierung der Lungenfunktion bei IPF-Patienten mit GER, die mit Antazida behandelt wurden [132]. Darüber hinaus beobachteten Lee et al. in einer präspezifizierten Analyse der IPFnet-Studien bei IPF-Patienten mit diagnostiziertem GER, die anschließend eine Antazida-Therapie (hauptsächlich Protonenpumpeninhibitoren (PPIs)) erhielten, eine langsamere Abnahme der Lungenfunktion [133]. Nach den aktualisierten evidenzbasierten IPF-Leitlinien von 2015 hat die geringe Qualität der Evidenzlage die Experten dazu veranlasst, eine bedingte Empfehlung für die Anwendung einer AntazidaTherapie bei IPF-Patienten zu geben [64]. Fidler et al. zeigten in einer kürzlich veröffentlichten systematischen Übersichtsarbeit und Meta-Analyse, darunter acht Beobachtungsstudien, dass eine medikamentöse GER-Therapie im Vergleich zur Nichtehandlung des GER mit einem signifikanten Rückgang der IPF-bezogenen Mortalität, jedoch nicht der Gesamtmortalität assoziiert war. Die Autoren wiesen jedoch auf die geringe Qualität der Evidenz für diese Outcomes hin und betonten die daraus resultierende Notwendigkeit randomisierter Studien, die den Effekt einer Antazida-Therapie bei IPF-Patienten untersuchen [134].

Eine kürzlich durchgeführte gepoolte Post-hoc-Analyse, die IPFPatienten aus den Placebo-Gruppen von drei Studien einschloss, in denen die Wirksamkeit und Sicherheit von Pirfenidon nachgewiesen wurden (die Studien CAPACITY 004, CAPACITY 006 und ASCEND), konnte die Effekte von PPIs dagegen nicht replizieren und berichtete über einen möglichen Zusammenhang mit einem erhöhten Infektionsrisiko bei Patienten mit fortgeschrittener Erkrankung [135]. In ähnlicher Weise lassen auch Post-hocAnalysen der INPULSIS-Studien, in denen die Sicherheit und Wirksamkeit von Nintedanib bei IPF-Patienten nachgewiesen wurde, vermuten, dass Patienten, die bei Baseline PPIs erhielten, möglicherweise schlechtere Ergebnisse erzielen [63]. Die oben genannten Post-hoc-Analysen sind unklar in Bezug auf die möglichen Vorteile einer Antazida-Therapie bei IPF-Patienten, und die spanischen [136] und deutschen [137] Leitlinien für IPF geben keine Empfehlung für eine Antazida-Therapie bei IPF-Patienten für ihre Grunderkrankung. Dies unterstreicht die dringende Notwendigkeit einer klar definierten, randomisierten, kontrollierten klinischen Studie, in der die Sicherheit und therapeutische Wirk- samkeit von PPIs/Antazida und Anti-Reflux-Operationen bei IPF prospektiv beurteilt werden.

Während Antazida-Therapien allein auf die Säure-Komponente abzielen, beinhaltet die chirurgische Intervention die Reparatur einer Hiatushernie und die Fundoplikatio nach Nissen zur Behandlung einer GERD durch Beseitigung der anatomischen Prädisposition für Reflux [138].

Diese Strategie wirkt dadurch, dass der Reflux von saurem und nicht saurem Mageninhalt aus dem Magen unterdrückt wird, wodurch das Aspirationsrisiko deutlich gesenkt wird [139]. Raghu et al. beobachteten einen möglichen Trend zur Stabilisierung der forcierten Vitalkapazität (forced vital capacity, FVC) vor und nach der laparoskopischen Anti-Reflux-Operation (laparoscopic anti-reflux surgery, LARS) über ein Jahr, was prospektive Studien erforderlich machte [140]. In einer ersten prospektiven und randomisierten klinischen Phase-2-Studie, der Studie Weighing Risks and Benefits of Laparoscopic Anti-Reflux Surgery in Patients with Idiopathic Pulmonary Fibrosis, in der die Sicherheit und Wirksamkeit der LARS-Eingriffe bei IPF-Patienten mit GERD untersucht wurden, konnten Raghu et al. zeigen, dass die LARS sicher und gut verträglich war. Obgleich gewisse klinisch bedeutsame Trends erkennbar waren, fand sich kein signifikanter Rückgang der Krankheitsprogression im Zeitverlauf. Atemwegsbezogene Hospitalisierungen und Todesfälle traten in der chirurgischen Gruppe seltener auf, jedoch ohne statistische Signifikanz [141].

\section{Obstruktive Schlafapnoe}

Verschiedene Studien ergaben, dass die obstruktive Schlafapnoe (OSA), die durch einen Apnoe-Hypopnoe-Index von >15 Ereignissen/Stunde definiert ist, bei IPF-Patienten häufig vorkommt $[142,143,144]$, und etwa 70\% dieser Patienten haben eine mittelschwere bis schwere OSA.

Verschiedene Veränderungen des Schlafes wurden bei IPF-Patienten festgestellt, darunter Veränderungen der Schlafarchitektur, wie beispielsweise eine abnorme Verteilung der Schlafphasen, mehrfaches Erwachen, ein verringerter Anteil an Gesamtschlafzeit, eine niedrige Schlafeffizienz und eine erhöhte Aufwachzeit nach Schlafbeginn, im Atemmuster, wie etwa erhöhte Atemfrequenz während des Schlafs und schnelle und flache Atmung, insbesondere während des REM (Rapid Eye Movement)-Schlafs, und bei den nächtlichen Oxygenierungsparametern, wie Sauerstoffentsättigung (sowohl während des REM- als auch während des Nicht-REM-Schlafs) und Entsättigung infolge respiratorischer Ereignisse (Apnoen und Hypopnoen) [145].

Das Spektrum an obstruktiven schlafbezogenen Atemstörungen bei IPF-Patienten umfasst eine Vielzahl respiratorischer Ereignisse, die während des Schlafs auftreten und von einfachem Schnarchen bis zum kompletten Aussetzen des Luftstroms (Apnoe) reichen [145]. Angesichts der hohen Prävalenz schlafbezogener Atemstörungen sollte zum Zeitpunkt der IPF-Diagnose eine nächtliche Polysomnographie erfolgen, um eine okkulte OSA festzustellen. Eine retrospektive Studie mit IPF-Patienten zeigte 
jedoch, dass bei weniger als 3\% der Patienten eine Polysomnographie durchgeführt wurde [142]. Die Bedeutung einer frühzeitigen OSA-Diagnose bei IPF-Patienten ergibt sich vor allem aus Hinweisen, nach denen eine unbehandelte OSA zu nächtlicher Hypoxämie führen kann, die sich als Prädiktor für eine schlechtere Überlebensdauer erwiesen hat [146].

Der vermutete Zusammenhang zwischen IPF und OSA beruht wahrscheinlich auf der Trachealzug-Theorie. Tatsächlich können die bei restriktiven Lungenerkrankungen beobachteten verminderten Lungenvolumina dazu führen, dass wegen der verringerten Traktion auf die oberen Atemwege die Stabilität der oberen Atemwege verringert wird und der Widerstand steigt, was einen Kollaps der oberen Atemwege begünstigt [145].

Patienten mit IPF und OSA sollten wie alle anderen OSA-Patienten eine Behandlung mit kontinuierlichem positivem Atemwegsdruck (continuous positive airway pressure, CPAP) erhalten, um die Lebensqualität zu verbessern und die Mortalität in diesem Patientenkollektiv zu senken [147].

Berichten zufolge ist allerdings die Rate an Nichtakzeptanz der CPAP-Therapie oder schlechter Compliance bei IPF-Patienten hoch, vor allem aufgrund von Klaustrophobie, Reizhusten während des Schlafs, Schlaflosigkeit und Depression, was zeigt, dass eine Verlaufskontrolle durch ein gut organisiertes Schlafzentrum erforderlich ist [148].

Zur Korrektur der nächtlichen Hypoxämie werden meist zusätzliche Sauerstoffgaben verordnet. Es gibt allerdings keine Studien, die einen Effekt auf das Langzeitüberleben oder auf die Entwicklung einer pulmonalen Hypertonie gezeigt haben [149].

\section{Lungenkrebs}

Das Risiko, an Lungenkrebs (lung cancer, LC) zu erkranken, ist bei Patienten mit IPF deutlich höher als in einer Kontrollpopulation [150]. Das erhöhte Risiko bleibt auch nach Adjustierung für Alter, Geschlecht und Rauchervorgeschichte bestehen, was vermuten lässt, dass die Lunge von Patienten mit IPF eine Prädisposition für die Entwicklung eines Malignoms aufweist. Interessanterweise steigt die kumulative Inzidenz von Lungenkrebs im Zeitverlauf deutlich an, von etwa 3,3\% ein Jahr nach der IPF-Diagnose auf 15,4\% nach 5 Jahren und 54,7\% nach 10 Jahren. In dieser Studie war das Alter bei Erstdiagnose der IPF ein signifikanter unabhängiger Faktor und Prädiktor für die Entwicklung von Lungenkrebs [151]. Die Mechanismen, die der Entwicklung von Lungenkrebs bei IPF zugrunde liegen, sind noch nicht vollständig geklärt. Neuere Hinweise scheinen jedoch dafür zu sprechen, dass möglicherweise eine gemeinsame genetische Prädisposition besteht. Bei Patienten mit IPF und Adenokarzinom der Lunge wurden Mutationen im Surfactant-Protein-A1 (SFTPA1)-Gen festgestellt, und bei den Angehörigen dieser Patienten war ein erhöhtes Risiko für die Entwicklung von interstitiellen Lungenerkrankungen oder anderen Formen von Krebs zu beobachten [152]. Interessant ist, dass der RAGE-Polymorphismus rs2070600 des Allels A, der bei schwerer IPF nachgewiesen wurde [80], möglicherweise mit einem erhöhten Lungenkrebsrisiko einhergeht [153].
Bei IPF-Patienten manifestiert sich Lungenkrebs häufig mit unspezifischen Symptomen, darunter Hämoptysen, Gewichtsverlust und andere Allgemeinsymptome. Daher sollte, wenn diese bei IPF-Patienten festgestellt werden, eine weitere Abklärung veranlasst werden [154]. Zudem kann die radiologische Diagnose bei diesen Patienten wegen der fibrotischen Veränderungen in der Lunge schwierig sein, da Lungenkrebs sich typischerweise in Form von knotigen Läsionen mit unregelmäßiger Begrenzung oder strahlenförmigen Ausläufern in peripheren Lungenbereichen darstellt, die schwer von fibrotischen Läsionen zu unterscheiden sind $[155,156]$. In einem Patientenkollektiv von italienischen IPF-Patienten waren periphere Plattenepithelkarzinome und Adenokarzinome die häufigsten histologischen Subtypen [157].

Zwar waren die Studien, die über Mortalität und Überlebensdauer von IPF-Patienten mit Lungenkrebs berichteten, durch kleine Stichprobengrößen eingeschränkt, doch zeigten sie, dass Patienten mit gleichzeitig vorliegendem LC eine kürzere Überlebensdauer aufwiesen als Patienten, die nur eine idiopathische interstitielle Pneumonie [158] oder IPF [157] hatten.

In der Regel sind IPF-Patienten mit Lungenkrebs keine guten Kandidaten für eine Standardtherapie, insbesondere, weil die Verträglichkeit von Krebstherapien bei diesen Patienten verringert ist. Daher sollte das Management von Lungenkrebs bei IPFPatienten einem individuellen Ansatz folgen, bei dem die Wahrscheinlichkeit des Behandlungserfolgs gegenüber potenziellen therapiebedingten Komorbiditäten abgewogen wird und der die oftmals schlechte Prognose bei IPF berücksichtigt [154]. Patienten mit Lungenfibrose weisen ein hohes Mortalitätsrisiko nach Thoraxoperationen, einschließlich Lungenresektion bei Lungenkrebs, auf [159]. Dieses erhöhte Risiko besteht häufig in einer sekundären AE-IPF, die bei etwa 10\% der IPF-Patienten nach thoraxchirurgischen Operationen auftritt und die in der Folge mit einer hohen Kurzzeitmortalität (ungefähr 50\%) verbunden ist [6]. Der genaue Mechanismus, über den chirurgische Eingriffe eine AE auslösen können, ist derzeit nicht bekannt.

Eine AE-IPF wurde auch bei Patienten beobachtet, die wegen Lungenkrebs eine Chemotherapie erhielten, oder nach einer Strahlentherapie $[160,161]$. Laut einer kürzlich veröffentlichten europäischen Umfrage zur Behandlung von Lungenkrebs bei Patienten mit IPF bevorzugten 54\% der Befragten die stereotaktische Strahlentherapie als Therapieansatz bei Patienten mit fortgeschrittener IPF (DLCO <35\%) und ansonsten operablem nicht kleinzelligem Lungenkarzinom. Platinhaltige Zweifach-Chemotherapie-Schemata und Immuntherapie bei metastasierter Erkrankung wurden von $25 \%$ bzw. 32\% der Befragten angegeben [162].

\section{Dekonditionierung und Sarkopenie}

Als Dekonditionierung wird ein Rückgang der physischen und/ oder psychischen Funktionsfähigkeit bezeichnet, der bei allen chronischen Erkrankungen häufig auftritt. Eine länger anhaltende Inaktivität oder verminderte Aktivität kann sich auf nahezu
240

Kompass Pneumol 2021;9:230-242

DOI: $10.1159 / 000518426$ 
alle Körpersysteme auswirken und kann als wichtige Komorbidität von chronischen Atemwegserkrankungen wie COPD angesehen werden [163]. Diese Veränderungen sind von großer Bedeutung, da sie die Fähigkeit zur Selbstversorgung, die Gehfähigkeit sowie die Fähigkeit die Freizeit zu gestalten und zu arbeiten beeinträchtigen können. Nishiyama et al. zeigten in ihrer prospektiven Studie, dass die körperliche Aktivität, ausgedrückt als zurückgelegte Schritte, bei Patienten mit IPF im Vergleich zu gesunden, nach Alter vergleichbaren Probanden signifikant reduziert war [164]. Die geringere tägliche körperliche Aktivität war zudem mit einer signifikant kürzeren Überlebensdauer dieser Patienten verbunden. Andere Autoren berichteten ähnliche Ergebnisse bei IPF [165] und anderen interstitiellen Lungenerkrankungen [166]. Die abnehmende funktionelle Kapazität ist einer der Hauptfaktoren für die schlechte Lebensqualität (quality of life, QoL) bei ILD/ IPF. Diese wird vor allem von der Belastungskapazität, der Lungenfunktion und der Dyspnoe beeinflusst [167]. Andere Komorbiditäten der IPF, wie KHK, COPD, PH, Verlust an Muskelmasse, Schmerzen und Depression, können ebenfalls einen Einfluss haben [168]. Ferner ist bekannt, dass die körperliche Leistungsfähigkeit von Patienten mit ILD durch zusätzliche Faktoren wie Durchblutungsstörungen und Funktionsstörungen der Beinmuskulatur eingeschränkt wird [169]. Die Dekonditionierung führt zu einem Verlust an Muskelmasse (Sarkopenie) und dieser wiederum zu einem Teufelskreis von reduzierter Aktivität [170].

Bei der Sarkopenie handelt es sich definitionsgemäß um einen fortschreitenden Verlust an Muskelmasse und -kraft, der mit zahlreichen negativen Folgeerscheinungen wie Behinderung, schlechter Lebensqualität und Tod verbunden ist [171]. Sie kann als Folge von Alterungsprozessen angesehen werden, die ein wichtiger Risikofaktor für IPF sind. Veränderungen der Körperzusammensetzung, die mit einem Schwund der Skelettmuskulatur, einem der Hauptfaktoren für pulmonale Kachexie, einhergehen, sind mit der Mortalität [172] bei COPD [173] und Krebs [174] assoziiert. Retrospektiven Studien zufolge kann eine geringe Muskelmasse bei IPF ein starker Risikofaktor für die Gesamtmortalität sein. In einer Studie mit Lungentransplantationspatienten war das Vorliegen einer ILD und COPD prädiktiv für die Muskelmasse vor und nach der Transplantation [175]. Die Sarkopenie steht mit der Gesamtmortalität, therapiebedingten Komorbiditäten, Lebensqualität und verminderter Atemmuskelkraft in Verbindung; allerdings sind diese Zusammenhänge bei IPF nicht gut untersucht.

Patienten mit dem komplexen klinischen Syndrom der Sarkopenie und Dekonditionierung, wie es bei IPF auftritt, profitieren eindeutig von multimodalen Interventionen, die einen auf die unterschiedlichen Phänotypen der Auszehrung zugeschnittenen therapeutischen Ansatz beinhalten. Eine frühzeitige Überweisung und Teilnahme an pulmonalen Rehabilitationsprogrammen kann eine wichtige Intervention zur Verbesserung der Symptome, der körperlichen Aktivität und der Lebensqualität bei IPF darstellen [176]. Vorläufige Daten lassen einen bescheidenen kurzfristigen Nutzen vermuten und es wird ein längerfristiges Erhaltungsprogramm empfohlen, um diese Erfolge zu bewahren [177]. Ver- haltensinterventionen, die soziale Unterstützung und eine Teilnahme an Selbsthilfegruppen umfassen, können die soziale Isolation vermindern und dazu beitragen, positive Veränderungen bei IPF zu verstärken.

\section{Malnutrition und Überernährung}

Nach den Ergebnissen mehrerer Studien scheinen Ernährungsanomalien, wie beispielsweise ein niedriger Body-Mass-Index (BMI), Gewichtsverlust und Vitamin-D-Mangel bei Patienten mit IPF ein negativer prognostischer Faktor zu sein [178]. Verschiedene Faktoren können negative Auswirkungen auf den Ernährungszustand von IPF-Patienten haben, darunter eine erhöhte Belastung der Atemmuskulatur, eine gleichzeitig bestehende Hypoxämie und körperliche Inaktivität, die zu Dynapenie (Abnahme der Muskelkraft) führt.

Trotz der Bedeutung von Ernährungsanomalien sind deren Prävalenz und Auswirkungen bei IPF größtenteils unzureichend erforscht.

Gemäß den in zahlreichen Studien angegebenen mittleren BMIWerten ist in den meisten Studien ein hoher Anteil an übergewichtigen bzw. adipösen Patienten zu erwarten [179, 180]. In fortgeschrittenen Krankheitsstadien können solche Störungen zu einem großen Problem werden, da Adipositas eine Kontraindikation für eine Transplantation darstellt und bei Patienten mit bilateraler Lungentransplantation mit einem erhöhten Mortalitätsrisiko assoziiert ist [8].

Zwar scheint die Prävalenz bei untergewichtigen Patienten geringer zu sein als bei übergewichtigen, doch fanden sich in einer kürzlich veröffentlichten Studie mit französischen IPF-Patienten bei fast einem Drittel der Fälle, insbesondere in fortgeschrittenen Krankheitsstadien, Veränderungen der Ernährungsparameter, die auf eine Malnutrition hindeuten [181].

Die beiden derzeit verfügbaren Antifibrotika Nintedanib und Pirfenidon können Nebenwirkungen verursachen, die die Nahrungsaufnahme und -resorption stören, darunter Durchfall, Übelkeit und Appetitlosigkeit [178].

Es sollte alles unternommen werden, um diese Nebenwirkungen zu reduzieren und so die Auswirkungen der antifibrotischen Langzeittherapien auf den Ernährungszustand zu minimieren.

\section{Welche Rolle spielen genetische Untersuchungen bei IPF?}

Die Bedeutung von Gentests bei IPF ist derzeit unklar und auf familiäre Formen der Lungenfibrose mit einer Prävalenz zwischen 2 und 20\% aller IPF-Fälle begrenzt [182, 183]. Es liegen zunehmend Hinweise vor, nach denen der Nachweis von pathogenen hTERT/ hTR-Mutationen klinisch von Bedeutung sein könnte, beispielsweise für das Outcome von Lungentransplantationsempfängern, die diese Mutationen tragen [184]. Außerdem könnten Gentests eine Antwort auf die Notwendigkeit präventiver Maßnahmen bei Geschwistern von Patienten mit pathogenen Mutationen sein; allerdings existieren keine Belege dafür, dass eine frühzeitige Diagnose und Präventionsmaßnahmen den Krankheitsverlauf verändern. 
Darüber hinaus besteht keine Einigkeit über das optimale Vorgehen bei der Abklärung von hTERT/hTR-Krankheits-assoziierten Varianten. Relativ einfache Assays wie die Flow-Fluoreszenz-insitu-Hybridisierung (Flow-FISH) oder die monochromatische quantitative Multiplex-Polymerase-Kettenreaktion (MM-qPCR) [185] können den Verdacht auf Mutationen bei familiären Formen durch Beurteilung der Telomerlänge erhärten; allerdings ist zur Bestätigung der Diagnose weiterhin eine Gensequenzierung erforderlich. Zunehmend sind in Gendiagnostiklabors Sequenzierungstechniken, einschließlich der Sequenzierung des gesamten Genoms (whole genome sequencing, WGS), verfügbar, und deren Kosten werden immer erschwinglicher. Durch Sequenzierung des gesamten Genoms könnten zudem potenziell mehrere Genmutationen bei demselben Patienten diagnostiziert werden, was dazu beitragen könnte, das sehr komplexe Zusammenspiel der an der Pathogenese der IPF beteiligten Gene zu beleuchten [186]. Bedauerlicherweise sind die klinische Bedeutung und der prädiktive Wert dieser Erkenntnisse unklar, so dass diese Tests nicht für die Versorgung von IPF-Patienten in der täglichen klinischen Praxis empfohlen werden können.

\section{Schlussfolgerungen}

Die IPF ist eine auf die Lunge begrenzte progrediente fibrosierende Erkrankung, und die zugelassenen antifibrotischen Therapien können den unaufhaltsamen Verlauf modifizieren, indem sie auf zahlreiche zelluläre Signalwege wirken. Darüber hinaus ist die IPF jedoch auch als eine Erkrankung anzusehen, die durch das Wechselspiel von Alterung, genetischer Prädisposition und Umweltfaktoren wie Zigarettenrauch entsteht, und die daher oft von Komorbiditäten begleitet wird, die den klinischen Phänotyp und das Überleben beeinflussen. Viele Komorbiditäten wirken sich sehr negativ auf verschiedene Outcome-Parameter bei IPF aus. Aus diesem Grund kann eine sorgfältige Diagnose und Behand- lung von Komorbiditäten eine ausgezeichnete Möglichkeit sein, das Überleben von Patienten mit IPF zu verbessern. Das breite Spektrum an Komorbiditäten bei IPF erfordert einen ganzheitlichen Ansatz, bei dem sämtliche infrage kommenden therapeutischen Optionen, einschließlich Früherkennung und Behandlung, berücksichtigt werden.

Es kann sein, dass die Aufklärung der feinen genetischen Mechanismen, die der IPF und ihren Komorbiditäten zugrunde liegen, zukünftig eine bessere Behandlung der Krankheit, insbesondere bei familiären Formen, ermöglichen wird.

\section{Interessenskonflikte}

Fabrizio Luppi hat persönliche Honorare von Roche und Boehringer-Ingelheim außerhalb der eingereichten Arbeit erhalten. Meena Kalluri hat persönliche Honorare von Roche und Boehringer-Ingelheim außerhalb der eingereichten Arbeit erhalten. Paola Faverio hat persönliche Honorare von Boehringer-Ingelheim außerhalb der eingereichten Arbeit erhalten. Michael Kreuter hat Forschungszuschüsse von Galapagos, Roche und Boehringer-Ingelheim erhalten. Er hat persönliche Honorare von Galapagos, Roche und Boehringer-Ingelheim außerhalb der eingereichten Arbeit erhalten. Giovanni Ferrara hat persönliche Honorare von Astra Zeneca, Roche und Boehringer-Ingelheim außerhalb der eingereichten Arbeit erhalten.

\section{Lizenzangabe}

Fabrizio Luppi, Meena Kalluri, Paola Faverio, Michael Kreuter, Giovanni Ferrara. Idiopathic pulmonary fibrosis beyond the lung: understanding disease mechanisms to improve diagnosis and management. Respir Res. 2021;22(1):109 (DOI: 10.1186/s12931-021-01711-1). ${ }^{\circ} 2021$ Die Autoren (Übersetzung; Publisher's Note, Verfügbarkeit der Daten und Materialien, Abkürzungen, Danksagung, Autorenbeiträge, Finanzierung der Studie, Verfügbarkeit der Daten und Materialien, Genehmigung durch die Ethikkommission und Einwilligung in die Teilnahme und Zustimmung zur Veröffentlichung gekürzt), lizensiert unter CC BY 4.0 (https://creativecommons.org/licenses/by/4.0/deed.de).

\section{Literatur}

Die Literatur ist unter www.karger.com/Article/Fulltext/518426 abrufbar. 